\title{
OCORRÊNCIA DE NEAR MISSES E FATORES ASSOCIADOS NA CLÍNICA CIRÚRGICA DE UM HOSPITAL DE ENSINO*
}

Thatianny Tanferri de Brito Paranaguá', Ana Lúcia Queiroz Bezerra², Ana Elisa Bauer de Camargo e Silva²

'Enfermeira. Mestre e Doutoranda em Enfermagem. Universidade Federal de Goiás. Goiânia-GO-Brasil.

${ }^{2}$ Enfermeira. Doutora em Enfermagem. Docente na Universidade Federal de Goiás. Goiânia-GO-Brasil.

RESUMO: Objetivou-se investigar a ocorrência e os fatores associados aos near misses relacionados à segurança de pacientes internados na clínica cirúrgica de um hospital de ensino. Estudo transversal, conduzido com 750 internações realizadas durante o ano de 2010, tendo os prontuários como fonte de dados. A coleta de dados ocorreu entre janeiro e maio de 2011. Realizada análise univariada e regressão logística. O registro do near miss ocorreu em 1,7\% das internações. Um total de 18 near misses foi registrado, sendo mais frequente o relacionado ao processo de prescrição de medicamentos. O sexo masculino foi associado como fator de proteção. Verificou-se a necessidade de estimular os profissionais de saúde ao registro dos near misses, a fim de subsidiar o processo de aprendizagem em serviço, permitir o estudo dos fatores causais e a promoção de melhorias no processo de trabalho.

DESCRITORES: Segurança do paciente; Erros médicos; Gestão da qualidade.

\section{THE OCCURRENCE OF NEAR MISSES AND ASSOCIATED FACTORS IN THE SURGICAL CLINIC OF A TEACHING HOSPITAL}

\begin{abstract}
This study aimed to investigate the occurrence of, and factors associated with, near misses related to the safety of patients undergoing inpatient treatment in the surgical clinic of a teaching hospital. It is a transversal study, undertaken with 750 episodes of inpatient treatment undertaken in 2010, using the medical records as the data source. Data collection occurred in January - May 2011. Univariate analysis and logistic regression were undertaken. The recording of near misses occurred in $1.7 \%$ of all the episodes of inpatient treatment. A total of 18 near misses was recorded, that related to the process of prescribing medications being the most frequent. Being male was associated as a protective factor. The need was ascertained to encourage health professionals to record near misses, so as to support the learning process at work, make it possible to study the causal factors, and promote improvements in the work process.

DESCRIPTORS: Patient safety; Medical errors; Management of quality.
\end{abstract}

\section{OCURRENCIA DE NEAR MISSES Y FACTORES ASOCIADOS EN LA CLÍNICA QUIRÚRGICA DE UN HOSPITAL DE ENSEÑANZA}

RESUMEN: Fue objetivo de este estudio investigar la ocurrencia y los factores asociados a los nearmisses referentes a la seguridad de pacientes internados en la clínica quirúrgica de un hospital de enseñanza. Estudio transversal, conducido con 750 internaciones realizadas durante el año de 2010, cuyos prontuarios fueron la fuente de datos. Los datos fueron obtenidos entre enero y mayo de 2011. Realizado análisis univariado y regresión logística. El registro del near miss ocurrió en 1,7\% de las internaciones. Un total de 18 near misses fue registrado, siendo más frecuente el relacionado al proceso de prescripción de remedios. El sexo masculino fue asociado como factor de protección. Se verificó la necesidad de estimular los profesionales de salud al registro de los near misses, con fines de subsidiar el proceso de aprendizaje en servicio, posibilitar el estudio de los factores causales y la promoción de mejorías en el proceso de trabajo.

DESCRIPTORES: Seguridad del paciente; Errores médicos; Gestión de la cualidad. 


\section{INTRODUÇÃO}

A responsabilidade médica e o aumento de incidentes com resultados negativos são temas frequentes na área da saúde, o que difundiu discussões acerca da segurança do paciente, mundialmente. Incidentes são definidos como qualquer ocorrência inesperada, durante a prestação de cuidados, que pode resultar em dano ao paciente. $\mathrm{O}$ incidente considerado mais grave é o evento adverso por, obrigatoriamente, resultar em algum tipo de dano ao paciente ${ }^{(1)}$.

Estudo transversal realizado em cinco países da América Latina estimou prevalência de $7 \%$ a $13 \%$ de eventos adversos ${ }^{(2)}$. No Rio de Janeiro, estudo de coorte realizado em três hospitais apontou, como principais eventos adversos evitáveis, as infecções associadas aos cuidados da saúde, que representaram $24,6 \%$ das ocorrências, seguido das complicações cirúrgicas e/ou anestésicas com 20,0\%; danos decorrentes do atraso ou falha no diagnóstico e/ou tratamento, $18,4 \%$ e úlceras por pressão, 18,4\%. Outros eventos adversos identificados foram associados à punção venosa, quedas e administração de medicamentos. Apontou ainda que esses eventos foram responsáveis por 373 dias adicionais de permanência no hospital (3).

No contexto cirúrgico, em decorrência das 234 milhões de cirurgias realizadas anualmente, estima-se que sete milhões de pacientes desenvolvem incidentes e um milhão vão ao óbito $^{(4)}$. Na América Latina, os eventos adversos cirúrgicos variam de 3,5\% a $16,4 \%{ }^{(2)}$, sendo $65,8 \%$ considerados evitáveis, segundo pesquisa nacional(5).

Após evidências de que o cuidado pode acarretar danos desnecessários ao paciente, é crescente o interesse das organizações de saúde em melhorar a segurança durante a provisão de cuidados. Nessa perspectiva, o estudo do near miss, que é definido pela Organização Mundial de Saúde (OMS) como incidente que, por sorte ou julgamento, foi interceptado antes de atingir o paciente ${ }^{(1)}$, tem sido apontado como indicador de avaliação do sistema organizacional, por indicar pontos falhos no processo de trabalho.

A análise prospectiva, transversal, de nove unidades de endoscopia evidenciou o registro de 230 incidentes, sendo $29 \%$ considerados eventos adversos e $71 \%$ considerados near misses. Dos 164 near misses analisados, 72\% auxiliaram na adoção de intervenções para correção e melhoria do processo de trabalho, reduzindo as chances de um erro atingir um paciente ${ }^{(6)}$.

Por representar um potencial evento adverso, a OMS incentiva a recuperação e monitoramento ativo do near miss pelas instituições de saúde ${ }^{(1)}$, entretanto, constitui-se em uma iniciativa ainda pouco observada e incipiente nas instituições de saúde. No intuito de revelar os riscos da assistência à saúde, subsidiar o estudo do near miss para a adoção de ações corretivas e evitar que esse potencial evento adverso resulte em dano, esse estudo objetiva investigar a ocorrência e fatores associados aos near misses relacionados à segurança de pacientes internados na clínica cirúrgica de um hospital de ensino.

\section{MÉTODO}

Estudo de corte-transversal, retrospectivo, tendo como fonte de dados os prontuários de pacientes internados na Clínica Cirúrgica de um hospital de ensino inserido na Rede Hospitais Sentinela da Agência Nacional de Vigilância Sanitária.

A escolha da clínica cirúrgica para a realização do estudo foi motivada pela experiência de pesquisadores no contexto cirúrgico, pelos profissionais terem recebido capacitação sobre incidentes e importância do registro de sua ocorrência e pelo hospital ter participado de estudos sobre cirurgias seguras, junto à Organização Pan-americana de Saúde.

A população do estudo foi composta por todos os prontuários de internações com data de admissão entre janeiro e dezembro de 2010, totalizando 2.610. Para determinar amostra representativa da população total foi utilizada a ferramenta de cálculo do OpenEpi, considerando os seguintes parâmetros: frequência hipotética de $50 \%$, pois não existem estudos que tratam da prevalência dos near misses no contexto da classificação da OMS; margem de erro de 5\%; efeito de desenho de 2,0 para garantir uma população robusta a ser pesquisada. Acrescentouse 80 prontuários em função de possíveis perdas relacionadas à captação dos dados, o que totalizou amostra de 750 prontuários, que foram selecionadas aleatoriamente, de forma probabilística e sistemática.

Foram incluídas as internações com tempo 
de permanência superior a 24 horas e aquelas com folha de prescrições e evolução. Todos os prontuários selecionados foram submetidos à avaliação para estimar a ocorrência de near misses em ambiente cirúrgico. A busca dos incidentes foi norteada por definição operacional, fundamentada na literatura científica e elaborada pelos próprios pesquisadores. A investigação ocorreu pela leitura minuciosa de todas as condutas da equipe multiprofissional registradas nas folhas de prescrição e evolução do paciente, desde a admissão até a alta hospitalar.

Os dados foram coletados entre janeiro e maio de 2011. Foi utilizado um formulário estruturado e pré-validado por estudo piloto, dividido em duas partes. A primeira parte continha questões para a investigação das características dos pacientes e aspectos da internação. A segunda parte investigava as características do incidente e continha espaço para a transcrição do registro identificado, para posterior análise e classificação dos incidentes.

As transcrições dos registros dos incidentes formaram o corpus de análise, sendo identificados pela letra $R$, seguido de número cardinal, conforme a ordem das internações. Após essa etapa, procedeu-se a análise dos incidentes identificados para a classificação dos near misses, que foi realizada por três pesquisadores, docentes e especialistas na temática segurança do paciente, com formação em enfermagem. O trabalho dos especialistas consistiu na análise e comparação do registro coletado com a definição de near miss proposta pela $\mathrm{OMS}^{(1)}$, na Classificação Internacional para a segurança do Paciente.

Os dados foram inseridos no software Statistical Package For The Social Science, versão 20.0 para Windows. Foi realizada análise descritiva dos dados e, para estimar os fatores associados ao near miss em análise univariada, utilizou-se quiquadrado e, quando necessário, teste exato de Fisher, com o OR (odds ratio) como medida de associação. Realizou-se análise multivariada de regressão logística com as variáveis: sexo, idade e as que obtiveram $p<0,10$ em análise univariada. Foram calculados intervalos de 95\% de confiança e consideradas, estatisticamente, significantes as associações que obtiveram valor de $p<0,05$.

Trabalho aprovado por Comitê de Ética, protocolo $\mathrm{N}^{\circ}$ 064/2008. Todas as normas nacionais e internacionais de ética em pesquisa envolvendo seres humanos foram atendidas.

\section{RESULTADOS}

O near miss foi registrado, no mínimo uma vez, em 1,7\% (13) das 750 internações. Foram registrados 18 near misses, correspondendo a 1,4 ocorrências por internação exposta, sendo $17(94,4 \%)$ casos relacionados à medicação e um (5,6\%) relacionado à intervenção cirúrgica.

A prescrição de medicamento para paciente alérgico correspondeu a 16 (88,9\%) near misses e, em todas as situações, a administração foi interceptada pelo próprio paciente que informava sua condição alérgica ao questionar o profissional sobre o nome do medicamento:

\section{0h. Paciente refere alergia a dipirona. (R12)}

22h. Antes de iniciar a administração de Dipirona, conforme prescrição, fui informada pelo paciente que ela era alérgica. (R351)

A principal conduta profissional foi destacar, na folha de prescrição, sobre a condição alérgica do paciente, seguida da solicitação de suspensão e substituição do medicamento. A categoria profissional responsável pelo registro de todos os near misses, relacionados à administração de medicamento para paciente alérgico, foi o técnico de enfermagem.

Verificou-se um (5,6\%) registro de near miss relacionado à super dosagem de contraste. $\mathrm{O}$ near miss foi interceptado pelos profissionais de outro setor de atendimento, na mesma instituição, ao preparar o paciente para um exame que necessitaria do uso de contraste. O exame foi suspenso após verificarem que, no mesmo dia, o paciente já havia recebido dose de contraste endovenoso:

15h. Paciente preparado e encaminhado para exame, mas foi suspenso devido ao paciente já ter realizado exame hoje, pela manhã, com uso de contraste. Remarco exame para próxima quarta-feira. (R122)

A conduta tomada frente ao near miss foi à remarcação do exame, que retardou o período de internação do paciente. Ressalta-se que o paciente foi preparado para o exame, foi 
deslocado da clínica de internação e ocupou o lugar de outros pacientes que, porventura, necessitariam do mesmo exame, interrompendo o plano de trabalho da unidade.

Quanto ao processo clínico verificou-se a ocorrência de um $(5,6 \%)$ caso de erro de diagnóstico que resultou em near miss relacionado à intervenção cirúrgica:

\section{8:30h. Cirurgia suspensa. Descarto lesão trófica e instituo tratamento clínico. (R75)}

Apesar de a cirurgia ter sido interceptada, o paciente passou por exames pré-operatórios, além de ter sido submetido à internação desnecessária e ao preparo cirúrgico.

A Tabela 1 apresenta a análise univariada relacionada à ocorrência dos near misses registrados nos prontuários dos pacientes.

$\mathrm{Na}$ análise univariada os valores de $\mathrm{p}<$ 0,05 apontaram diferença estatística para as variáveis: sexo masculino, tempo de internação igual ou superior a quatro dias, prescrição de três ou mais medicamentos por dia, utilização de sonda vesical e infusão de hemoderivados, evidenciando pacientes com características de maior probabilidade em se expor ao near miss.

Para verificar o comportamento dessas variáveis como fator independente para a ocorrência de near miss, as mesmas foram submetidas à análise de regressão logística, conforme o disposto na Tabela 2.

A regressão logística evidenciou que a única variável independente associada à ocorrência do near miss foi o sexo masculino que se comportou como fator de proteção, indicando menor probabilidade de ocorrência do near miss em relação ao sexo feminino.
Tabela 1 - Associação univariada a entre as variáveis de exposição e a ocorrência de near miss, durante internações na Clínica Cirúrgica de um hospital de ensino. Goiânia-GO, 2011.

\begin{tabular}{|c|c|c|c|}
\hline Variáveis & $\begin{array}{c}n \\
\text { expostos/total }\end{array}$ & $\%$ & $\mathbf{p}$ \\
\hline \multicolumn{3}{|l|}{ Idade em anos } & \multirow{3}{*}{0,781} \\
\hline Até 45 & $7 / 351$ & 2,0 & \\
\hline Acima de 46 & $6 / 399$ & 1,5 & \\
\hline \multicolumn{3}{|l|}{ Sexo } & \multirow{3}{*}{$0,016^{*}$} \\
\hline Masculino & $1 / 301$ & 0,3 & \\
\hline Feminino & $12 / 449$ & 2,7 & \\
\hline \multicolumn{3}{|l|}{ Comorbidade } & \multirow{3}{*}{0,141} \\
\hline Sim & $8 / 312$ & 2,6 & \\
\hline Não & $5 / 438$ & 1,1 & \\
\hline \multicolumn{3}{|c|}{ Tipo de admissão } & \multirow{3}{*}{0,264} \\
\hline Urgência & $4 / 134$ & 3,0 & \\
\hline Eletiva & $9 / 616$ & 1,5 & \\
\hline
\end{tabular}

Tempo de internação em dias

\begin{tabular}{|c|c|c|c|}
\hline 4 ou mais & $10 / 329$ & 3,0 & $0,015^{*}$ \\
\hline 1 à 3 & $3 / 421$ & 0,7 & \\
\hline
\end{tabular}

Total de medicamentos prescritos por dia

\begin{tabular}{|c|c|c|c|}
\hline 3 ou mais & $10 / 325$ & 3,1 & $0,014^{*}$ \\
\hline Até 2 & $3 / 425$ & 0,7 & \\
\hline \multicolumn{3}{|c|}{ Intervenção cirúrgica } & \multirow{3}{*}{0,098} \\
\hline Sim & $9 / 644$ & 1,4 & \\
\hline Não & $4 / 106$ & 3,8 & \\
\hline \multicolumn{3}{|c|}{ Profilaxia antibiótica } & \multirow{3}{*}{0,565} \\
\hline Não & $6 / 277$ & 2,2 & \\
\hline Sim & $7 / 473$ & 1,5 & \\
\hline \multicolumn{3}{|c|}{ Utilização de sonda vesical } & \multirow{3}{*}{ 0,030* } \\
\hline Sim & $8 / 235$ & 3,4 & \\
\hline Não & $5 / 515$ & 1,0 & \\
\hline
\end{tabular}

Utilização de dreno de penrose

\begin{tabular}{|c|c|c|c|}
\hline Sim & $2 / 107$ & 1,9 & 1,000 \\
\hline Não & $11 / 643$ & 1,7 & \\
\hline
\end{tabular}

Utilização de cateter venoso

\begin{tabular}{|c|c|c|c|}
\hline Sim & $12 / 680$ & 1,8 & 1,000 \\
\hline Não & $1 / 70$ & 1,4 & \\
\hline \multicolumn{3}{|c|}{ Infusão de hemoderivados } & \multirow{3}{*}{$0,017^{*}$} \\
\hline Sim & $4 / 62$ & 6,5 & \\
\hline Não & 9/688 & 1,3 & \\
\hline
\end{tabular}

*Significância estatística, pois $\mathrm{p}<0,05$. Obtido a partir do teste $\mathrm{x} 2$. 
Tabela 2 - Regressão logística e medida de associação (Odds Ratio) das variáveis de exposição ao near miss, com $\mathrm{p}<0,05$ obtido em análise univariada, ocorrido durante internações na Clínica Cirúrgica de um hospital de ensino. Goiânia-GO, 2011.

\begin{tabular}{lcc}
\hline Fatores de risco para near miss & Odds ratio (ic 95\%) & P \\
\hline Idade $>45$ anos & $1,8(0,6-5,8)$ & 0,311 \\
\hline Sexo masculino & $0,1(0,0-0,9)$ & $0,039^{*}$ \\
\hline Tempo de internação $>4$ dias & $1,5(0,3-7,2)$ & 0,638 \\
\hline Medicamentos prescritos $>$ 3/dia & $2,3(0,5-10,6)$ & 0,290 \\
\hline Realizar intervenção cirúrgica & $0,3(0,1-1,1)$ & 0,065 \\
\hline Utilizar sonda & $3,1(0,8-12,4)$ & 0,115 \\
\hline Infundir hemoderivado & $2,6(0,6-10,6)$ & 0,199 \\
\hline
\end{tabular}

*Significância estatística, pois $\mathrm{p}<0,05$. Obtido a partir do teste $\mathrm{x} 2$.

\section{DISCUSSÃO}

A ocorrência do near miss foi de 1,7\%. O desenvolvimento de uma cultura de registro desses indicadores deve ser estimulado, considerando as recomendações da OMS para a detecção de falhas latentes, direcionando a alocação de recursos para ações preventivas.

James Reason ${ }^{(7)}$ defende que o sistema de saúde é comparado a um queijo suíço. Os furos representam as falhas latentes do processo de trabalho e que, se não controladas e/ou sanadas, podem resultar em uma falha ativa, ou seja, um incidente que atinge o paciente, portanto, o estudo do near miss pode contribuir com a identificação de lacunas, refletir no aumento das chances de êxito das instituições de saúde ao lidar com atos inseguros e permitir o aprimoramento contínuo do processo de trabalho.

Foram identificados dois tipos de near misses que evidenciaram a característica multifatorial e a dimensão sistemática nos quais estão inseridos. Aqueles relacionados à medicação indicaram a necessidade de modificar o processo de prescrição de medicamentos, especialmente, no fator envolvimento do paciente durante a prescrição, uma vez que esta deve ser precedida de investigação sobre medicamentos em uso contínuo e antecedentes alérgicos.

Os erros de prescrição podem variar de $24,0 \%$ a 29,3\%, dependendo das ações adotadas pela instituição de saúde. Entretanto, a redução de sua ocorrência não influencia a gravidade das consequências ao paciente ${ }^{(8)}$. Estudo transversal realizado em hospital de ensino verificou que do total de incidentes relacionados à medicação, $4,1 \%$ se referiram aos erros de prescrição, os quais foram classificados como near misses, uma vez que foram interceptados pela equipe de enfermagem na fase de administração ${ }^{(9)}$.

O conhecimento da equipe de enfermagem sobre os medicamentos é fundamental para a construção de uma barreira de segurança ao paciente, que pode ser mais efetiva quando aliada à prescrição eletrônica e ao farmacêutico clínico $^{(10)}$.

Estudo que analisou o processo de administração de medicamentos de um hospital escola evidenciou que $43,9 \%$ das falhas do sistema estão relacionadas à gestão dos processos organizacionais, como sobrecarga de trabalho e ausência de rotinas e de educação continuada; $41,4 \%$ se relacionam aos recursos humanos, como déficit de conhecimento, habilidades e falta de atenção e 12,6\% evidenciam necessidades relacionadas à estrutura física e material(11).

Dentre os fatores associados à ocorrência de incidentes com medicamentos estão o tempo de internação superior a quatro dias, a prescrição de quatro ou mais medicamentos por dia e a realização intervenção cirúrgica ${ }^{(12)}$, indicando a necessidade de dispensar maior atenção a esses pacientes. $\mathrm{O}$ uso de ferramentas de análise de risco, como o Failure Mode and Effect Analysis (FMEA), tem se mostrado efetivo para o gerenciamento de falhas no processo de medicação, auxiliando no direcionamento de ações de melhoria e segurança ${ }^{(11)}$.

Outra ferramenta para a redução dos incidentes e que foi evidenciada na maioria dos near misses, relacionados ao processo de medicação identificados nesse estudo, é a participação do próprio paciente nas ações de cuidados. O cuidado centrado no paciente e 
em sua família é uma das principais dimensões a ser enfatizada no sistema de saúde, para se alcançar qualidade ${ }^{(13)}$. O paciente, portanto, é visto como sujeito ativo no processo saúdedoença, contribuindo para um tratamento mais efetivo, apropriado e seguro ${ }^{(14-15)}$.

A importância de manter adequada comunicação entre os diferentes setores de uma organização hospitalar foi evidenciada ao interceptar o near miss relacionado à administração de contraste.

Estudo que analisou os near misses vivenciados por enfermeiras, em ambiente perioperatório nos Estados Unidos, verificou que a falha de comunicação entre a equipe de saúde constitui a principal causa dos near misses ${ }^{(16)}$. Transmitir informações sobre o paciente em ambiente tranquilo e sem interrupções, registrar as condições do paciente, os medicamentos necessários, os resultados de exames, a previsão do tratamento, as recomendações sobre os cuidados, as alterações significativas e os procedimentos realizados são algumas orientações para se estabelecer uma comunicação efetiva e segura, especialmente, entre os setores que o paciente tramita durante a internação ${ }^{(17-18)}$.

Falta de formação e de treinamento dos profissionais de saúde são reconhecidos como os principais fatores que influenciam a qualidade da comunicação, o que tem motivado a alteração curricular dos cursos na área da saúde. O desenvolvimento de competências comunicacionais deve ser incluído na formação e, assim como acontece com outras competências de ordem técnica e clínica, precisam ser, sistematicamente, atualizadas ${ }^{(19)}$.

O near miss relacionado à intervenção cirúrgica foi decorrente de um erro de diagnóstico. A análise dos processos registrados no Conselho Regional de Medicina do Estado de Goiás evidenciou que $10,3 \%$ dos casos denunciados por incompetência médica são correspondem ao erro diagnóstico ${ }^{(20)}$. É importante ao profissional atentar-se ao significado da relação médicopaciente, especialmente, quando se diz respeito à comunicação e ao estabelecimento de um modelo de medicina mais humanístico, visando ao respeito e à dignidade do paciente, proporcionando possibilidades para melhor investigação dos casos e evitando, portanto, uma série de incômodos e aborrecimentos ${ }^{(19-20)}$.
A adequada investigação da situação de saúde do paciente e o conhecimento científico possibilitam a redução de preparo cirúrgico desnecessário e, principalmente, de diagnósticos equivocados. Fortalecer a integração das ações entre as diversas categorias profissionais podem minimizar os potenciais riscos de uma internação e aumentar as possibilidades de detecção de near misses para que um incidente grave não aconteça.

Estudo transversal conduzido com 427 profissionais, incluindo médicos, enfermeiros e anestesistas, evidenciou outros problemas na sala de cirurgia. Dentre os profissionais, 38\% referiram ter vivenciado near misses relacionados à identificação do paciente, $60 \%$ ao preparo do paciente para procedimento e $81 \%$ ao local ou lado da cirurgia(21).

O sexo masculino foi fator de proteção para a ocorrência do near miss, o que difere de outros estudos que apontam maior probabilidade de ocorrência de incidentes em pacientes do gênero masculino $^{(22)}$.

A utilização de sonda vesical e a infusão de hemoderivados não apontaram associação para a ocorrência de near misses. Comumente, o uso desses dispositivos está associado à condição clínica e à gravidade do paciente e remete ao aumento dos riscos clínicos, do número de intervenções durante a internação e do tempo dispendido pelos profissionais ${ }^{(23)}$. Além disso, vários são os estudos que apontam o uso de dispositivos tubulares como aumento do risco da ocorrência de incidentes e orientam cuidados específicos para sua prevenção ${ }^{(24)}$.

A transfusão de sangue, quando realizada de forma insegura, pode estar associada à reação transfusional, transmissão de infecção, aumento de morbidade e mortalidade pós-operatória e risco de imunossupressão ${ }^{(25-26)}$, o que exige maior atenção do profissional.

Diante do cenário atual, portanto, evidenciase a necessidade de estudar os near misses de modo a evitar que potenciais eventos adversos aconteçam e comprometam, ainda mais, a assistência e a segurança do paciente.

Nos Estados Unidos da América, o National Fire Fighter Near Miss Reporting System é uma iniciativa coordenada pela International Association of Fire Chiefs e pela Escola de Saúde Pública da Universidade de Drexel, que utiliza a web para a construção de um banco de dados 
para estudos de near misses e tem a finalidade de alertar os bombeiros acerca dos perigos em serviço. A notificação é voluntária, confidencial, segue um sistema de informação não punitivo e seguro e os near misses são utilizados como ferramentas de aprendizagem ${ }^{(27)}$.

No contexto da saúde verifica-se escassez de literatura que descreve experiências que reportem o near miss, especificamente, para estudo das falhas e tomada de ações preventivas. Ainda prevalecem os estudos que tratam de incidentes mais graves, que necessitam de intervenções corretivas imediatas e que, na maioria das situações, causaram dano ao paciente.

A Portaria $N^{\circ} 529$ de $1^{\circ}$ de abril de 2013, do Ministério da Saúde, que institui o Programa Nacional de Segurança ${ }^{(28)}$, poderá estimular o interesse de gestores e profissionais de saúde para a adesão de conhecimentos sobre os incidentes, além de desenvolver a cultura de segurança do paciente nas instituições de formação e assistência em saúde.

Entretanto, acredita-se que para uma aprendizagem significativa em serviço é necessário, primeiramente, sistematizar o processo de monitoramento dos incidentes e near misses decorrentes da assistência à saúde. Torna-se, portanto, desafio para os gestores de saúde fortalecer as comissões de gerenciamento de risco, envolver os profissionais de saúde na busca ativa de falhas latentes e os sensibilizar para a mudança de atitude, com ênfase na adoção de boas práticas e na promoção de um sistema de resiliência sistemático, contribuindo continuamente para a assistência de qualidade e excelência.

\section{CONCLUSÃO}

Constatou-se a ocorrência de 1,7\% de near misses que indicaram falhas no processo de administração de medicamento, na comunicação intersetorial e na avaliação clínica do paciente para julgamento de diagnóstico médico, as quais se configuram em potenciais eventos adversos, caso não sejam monitoradas e discutidas pelo serviço de saúde.

O estudo apresenta limitações quanto ao método utilizado e pode não refletir o número real de near misses ocorridos, uma vez que o registro dos incidentes depende da atitude do profissional de saúde e da cultura organizacional da instituição.
Constata-se, portanto, a necessidade de estimular os profissionais de saúde para o registro dos near misses, a fim de permitir o estudo dos fatores causais e a promoção de mudanças na organização do trabalho em saúde. Sugere-se a educação em serviço para despertar e desenvolver o profissional para a cultura de segurança e, ainda, a adoção de instrumentos específicos para o registro dos near misses, de modo a facilitar seu rastreamento e formar um banco de indicadores para análise e direcionamento dos processos de trabalhos, possibilitando a redução das chances de um incidente resultar dano ao paciente.

\section{REFERÊNCIAS}

1. WHO - World Health Organization. Conceptual framework for the international classification for patient safety. In: Conceptual Framework for the International Classification for Patient Safety. Final Technical Report. [Internet] 2009 [cited $19 \mathrm{dez}$ 2010]. Avaliable from: http://www.who.int/patientsafety/taxonomy/icps_full_ report.pdf

2. Ministerio de Sanidad y Politica Social. Estudio IBEAS: Prevalencia de efectos adversos en hospitales de Latinoamérica. [Internet] 2010 [acesso em $15 \mathrm{dez}$ 2013]. Disponível: www.msssi.gob.es/organizacion/ sns/planCalidadSNS/docs/INFORME_IBEAS.pdf

3. Mendes W, Pavão ALB, Martins M, Moura MLO, Travassos C. Características de eventos adversos evitáveis em hospitais do Rio de Janeiro. Rev. Assoc. Med. Bras. 2013;59(5):421-8.

4. OMS - Organização Mundial da Saúde. Segundo desafio global para a segurança do paciente: manual - cirurgias seguras salvam vidas (orientações para cirurgia segura da OMS). Trad. de Marcela Sánchez Nilo e Irma Angélica Durán. Rio de Janeiro (Brasil): Organização Pan-Americana da Saúde; 2009.

5. Moura MLO, Mendes W. Avaliação de eventos adversos cirúrgicos em hospitais do Rio de Janeiro. Rev. Bras. Epidemiol. 2012;15(3):523-35.

6. Minoli G, Borsato P, Colombo E, Bortoli A, Casetti $\mathrm{T}$, Pretis $\mathrm{G}$, et al. Errors and near misses in digestive endoscopy units. Digestive and Liver Disease. 2012; 44(11): 914-8.

7. Reason J. El error humano. Madrid: Modus Laborandi; 2009.

8. Néri EDR, Gadêlha PGC, Maia SG, Pereira AGS, Almeida PC, Rodrigues CRM, et al. Erros de prescrição de medicamentos em um hospital brasileiro. Rev. Assoc. Med. Bras. 2011;57(3):306-14. 
9. Teixeira TCA, Cassiani SHB. Análise de causa raiz: avaliação de erros de medicação em um hospital universitário. Rev. Esc. Enferm. USP. [Internet] 2010;44(1) [acesso em 16 dez 2013]. Disponível: http:// dx.doi.org/10.1590/S0080-62342010000100020

10. Charpiat B, Goutelle S, Schoeffler M, Aubrun F, Viale JP, Ducerf $C$, et al. Prescriptions analysis by clinical pharmacists in the post-operative period: a 4-year prospective study. Acta anaesthesiol. scand. 2012;56:1047-51.

11. Silva AEBC, Cassiani SHB. Análise prospectiva de risco do processo de administração de medicamentos antiinfecciosos. Rev. Latino-Am. Enfermagem. [Internet] 2013;21 [acesso em 30 nov 2014]. Disponível: http:// dx.doi.org/10.1590/S0104-11692013000700029

12. Paranaguá TTB, Bezerra ALQ, Santos ALM, Silva AEBC. Prevalence and factors associated with incidents related to medication in surgical patients. Rev. Esc. Enferm. USP. [Internet] 2014;48(1) [cited 10 nov 2014]. Avaliable from: http://dx.doi.org/10.1590/S0080623420140000100005

13. Pedreira MLG. Enfermagem para a segurança do paciente. Acta Paul. Enferm. 2009;22(4): V-VI.

14. Mitchell PH. Patient-centered care: a new focus in a time honored concept. Nurs Outlook. 2008;56(5): 97-8.

15. Vincent C. Segurança do paciente: orientações para evitar eventos adversos. São Caetano do Sul: Yendis; 2009.

16. Cohoon B. Causes of near misses: perceptions of perioperative nurses. AORN j. 2011;93(5):551-65.

17. Avelar AFM, Salles CLS, Bohomol E, Feldman LM, Peterlini MAS, Harada MJCS, et al. Dez passos para a segurança do paciente. Conselho Regional de Enfermagem do Estado de São Paulo. Rede Brasileira de Enfermagem e Segurança do Paciente. São Paulo, 2010.

18. Padilha EF, Haddad MCFL, Matsuda LM. Qualidade dos registros de enfermagem em terapia intensiva: avaliação por meio da auditoria retrospectiva. Cogitare enferm. 2014;19(2):239-45.

19. Santos MC, Grilo A, Andrade G, Guimarães T, Gomes A. Comunicação em saúde e a segurança do doente: problemas e desafios. Rev. port. saude publica. 2010;10:47-57.

20. Fujita RR, Santos IC. Denúncias por erro médico em Goiás. Rev. Assoc. Med. Bras 2009;55(3):283-9.

21. Haugen AS, Murugesh S, Haaverstad R, Eide GE, Softeland E. A survey of surgical team members' perceptions of near misses and attitudes towards Time
Out protocols. BMC Surgery. 2013;13(46):1-7.

22. Roque KE, Melo ECP. Tempo de internação e a ocorrência de eventos adversos a medicamentos: uma questão da enfermagem. Esc. Anna Nery. [Internet]. 2011;15(3). [acesso em 11 dez 2013]; Disponível: http:// dx.doi.org/10.1590/S1414-81452011000300022

23. Alghamdi AA, Davis A, Brister S, Corey P, Logan A. Development and validation of transfusion risk understanding scoring tool (TRUST) to stratify cardiac surgery patients according to their blood transfusion needs. Transfusion. 2006;46(7):1120-9.

24. Silva AEBC, Laselva CR, Carrara D, Perini E, Pinto GRS, Sousa MRG et al. Erros de conexão: práticas seguras e riscos na administração de soluções por sondas enterais e cateteres vasculares. Boletim ISMP. 2013;2(3):1-3.

25. Banbury MK, Brizzio ME, Rajeswaran J, Lytle BW, Blackstone $\mathrm{EH}$. Transfusion increases the risk of postoperative infection after cardiovascular surgery. J Am Coll Surg. 2006;202(1):131-8.

26. Souza HJB, Moitinho RF. Estratégias para redução do uso de hemoderivados em cirurgia cardiovascular. Rev Bras Cir Cardiovasc. 2008;23(1):53-9.

27. National Fire Fighter Near-Miss Reporting System [Internet]. 2012. [cited 19 jul 2013]. Avaliable from: http://www.firefighternearmiss.com/

28. Ministério da Saúde. Portaria $N^{\circ} 529$, de $1^{\circ}$ de abril de 2013. Institui o Programa Nacional de Segurança do Paciente. Brasília; 2013. 